\title{
GESTIÓN DEL TALENTO DE LOS EMPLEADOS PÚBLICOS DEL SECTOR TURÍSTICO EN ANDALUCÍA
}

\author{
José Luis Sánchez-Ollero* \\ Universidad de Málaga \\ https://orcid.org/0000-0003-0834-8493 \\ Francisco Sánchez-Cubo** \\ Universidad de Castilla La-Mancha \\ https://orcid.org/0000-0002-0556-7239 \\ Elisa del Cubo-Arroyo*** \\ Universidad de Málaga \\ https://orcid.org/0000-0003-4589-7256
}

\section{RESUMEN}

La gestión del talento del personal de la Administración Pública del sector turístico es un gran reto. Sobre todo, por la necesidad de dar soluciones a la falta de flexibilidad de sus RRHH en un sector caracterizado por su dinamismo y especialización.

En este trabajo se analiza la situación actual de los empleados públicos en Andalucía, se valida un cuestionario sobre la percepción de sus trabajadores acerca del talento y se desarrolla una herramienta innovadora sobre la Gestión Estratégica del Talento, apoyada en la "Teoría del Valor", que podría aumentar la productividad y satisfacción entre sus trabajadores.

Palabras clave: Empleado del Sector Público; Gestión del Talento; Reclutamiento de Talento; Retención del Talento; Sector Turístico.

Talent management of public employees in the tourist sector in Andalusia

Fecha de recepción: 10 de septiembre de 2019.

Fecha de aceptación: 1 de septiembre de 2020.

* Departamento de Economía Aplicada (Estructura Económica). Facultad de Turismo. Universidad de Málaga. Campus de Teatinos.29071MÁLAGA (España).E-mail: jlsanchez@uma.es

** Facultad de Ciencias Sociales de Cuenca. Universidad de Castilla-La Mancha. Avda. de los Alfares, 44. 16071 CUENCA (España).E-mail: francisco.scubo@uclm.es

*** Área de Investigación. Edificio Institutos de Investigación. Universidad de Málaga. Campus de Teatinos.29071 MÁLAGA (España).E-mail: eidelcubo@uma.es 


\begin{abstract}
Managing Public Sector Employees' Talent in the Tourism Sector is a huge challenge, especially due to the need to find solutions to the lack of flexibility of its HR in such a dynamic and specialised sector.

This paper analyses the current situation of the Andalusian's public employees, is validated a survey about these employees' perception about talent and it is also developed an innovative tool about Strategic Talent Management, which is based on the 'Theory of Value', that may increase job performance and job satisfaction between its employees.
\end{abstract}

Keywords: Public Sector Employee; Talent Management; Talent Recruitment; Talent Retention, Tourism Sector.

\title{
1. INTRODUCCIÓN
}

El turismo es bien conocido por ser uno de los motores principales de la economía global (10,4\% del PIB mundial en el año 2017) (World Travel \& Tourism Council, 2018) siendo, en países como España, donde supone un 11,7\% del PIB nacional (Instituto Nacional de Estadística, 2018), una actividad de vital importancia para el desarrollo de regiones carentes de otros factores productivos tales como industrias o centros de I+D+i. En este sentido, la región de Andalucía, unidad territorial y política tomada para la realización de este trabajo, es un claro ejemplo de zona beneficiada por el impacto del turismo sobre otras actividades, pues éste supone unos ingresos de 20,6 mil millones de euros, es decir, un 12,8\% del PIB regional (Consejería de Turismo y Deporte, 2017).

Así, dado el importante peso de este sector en la economía, es crucial una participación más activa, eficaz y sincronizada de todos los agentes públicos y privados involucrados, con el fin de consolidar la actividad turística en entornos cada vez más competitivos. Un contexto en el que los consumidores se encuentran cada vez más y mejor informados, son más exigentes y, además, buscan experiencias más novedosas (Cuadrado-Roura y López-Morales, 2014) que no sólo cubran sus deseos, sino que superen sus expectativas. Este escenario pone de manifiesto la importancia de la gestión de los recursos humanos como eje estratégico que impulse la competitividad, la eficacia y actúe como elemento diferenciador entre los distintos servicios que ofrece este mercado.

Además, la innovación mediante la implementación de las nuevas tecnologías en el sector turístico (el uso del Big Data, la aplicación de la realidad virtual y aumentada en museos y sitios de interés, la fuerte dependencia de las redes sociales, etc.), ha dado lugar a la necesidad de adaptación a este nuevo contexto digital. Por tanto, es indispensable dar respuesta a las exigencias digitales de la demanda, no solo en el proceso de información y adquisición de productos turísticos, también durante su disfrute para generar experiencias auténticas (Sundbo, 2009).

En consecuencia, se antoja imprescindible disponer de personas versátiles, transversales, capaces no sólo de convivir con la inmediatez y la exigencia del mercado turístico, 
sino de adaptarse continuamente. Este panorama no solo afecta a las plantillas básicas, sino a toda la jerarquía empresarial (tanto privada como pública) e institucional, cobrando especial relevancia la figura de los directivos para potenciar el talento de sus trabajadores dentro del marco estratégico de la empresa/institución. Por tanto, es en este punto donde debe hacerse uno de los mayores esfuerzos: generar y retener el talento de sus recursos humanos como activo de valor de la entidad.

En este sentido, el ámbito público está poco estudiado a pesar de su importancia en el sector del turismo como organismo regulador, pero también como agente activo en la gestión del desarrollo de la actividad turística en su territorio. Es por ello, este texto persigue conocer la estructura de los empleados públicos del sector turístico en Andalucía, así como su sistema de acceso y promoción para analizar y proponer una herramienta, en torno a su marco normativo, innovadora y aplicativa de la gestión del talento. Entendemos esencial la explotación de sus capacidades en pos del éxito turístico del territorio andaluz, aunque se caractericen por la rigidez tanto de su estructura como de sus procedimientos, algo claramente inverso a lo que exige el contexto turístico actual. Además, esta línea de investigación se presenta novedosa, pues la vasta literatura académica en relación con la gestión del talento es escasa en el entorno público.

El presente trabajo se estructura, en primer lugar, en el análisis de las diferentes estrategias sobre recursos humanos utilizadas en el sector público. En segundo lugar, se discuten las definiciones de "Talento" y "Gestión del talento" en base a la revisión de otros trabajos que aplican este tipo de gestión y una exhaustiva revisión de la literatura existente sobre Gestión del Talento y Turismo. Además, se explica la llamada "Teoría del valor" que respalda las estrategias propuestas, exponiendo casos de estudio que ayuden a contextualizar el alcance de la teoría. En tercer lugar, se proporciona una descripción del marco contractual de los empleados del sector público en general. En el siguiente apartado, caso de estudio, se estudia específicamente el sector turístico en Andalucía, que incluye una aproximación al número de empleados que trabajan en él.

A continuación, se explica el pretest empleado: metodología, validación y análisis de los resultados para, finalmente, proponer una herramienta innovadora de Gestión Estratégica del Talento con el fin de establecer una vía de adaptación y flexibilización de la Administración Pública Andaluza en materia de turismo donde el acceso, la promoción y la retención se presentan como aspectos clave.

\section{MARCO CONCEPTUAL}

La gestión de los recursos humanos de la Función Pública forma parte de la implantación de: 1) modelos normalizados, importados de la empresa privada, que gestionan la calidad en su conjunto, Modelo EFQM o Modelo de Calidad Total, con el objeto de alcanzar unos estándares en materia de personal adaptados a las necesidades de cada administración; 2) la implementación de modelos teóricos que surgen del estudio de especialistas de lo público que proporciona un sistema integrado de gestión, cuya finalidad básica es la adecuación de las personas a la estrategia de cada organización pública (Longo, 2004) y 3) el ajuste de estrategias específicas de recompensas (Thompson, 2002), mejora del liderazgo (Blanco, 2016), gestión del conocimiento y desarrollo de 
competencias (Instituto Andaluz de Administración Pública, 2008) y mejora del desempeño (Boletín Oficial del Estado, 2015).

Todas las alternativas aportan resultados parciales en función del sector institucional y la finalidad organizativa, dando por sentado la naturaleza heterogénea de la Administración Pública nacional. Además, esta estrategia ni pone en valor la Función Pública como institución de servicio público ni pretende modificar un sistema de reclutamiento basado en pruebas memorísticas en vez de la evaluación de competencias y habilidades destinadas a interactuar con los nuevos retos sociales: inteligencia artificial, Big Data y trabajo a distancia.

Por ello, consideramos necesario utilizar un instrumento global que planifique cómo quiere ser valorada la organización, a quiénes atraer, cómo motivarlos y protegerlos. En definitiva, cómo gestionar el talento para aumentar la calidad en el rendimiento del servicio público para el bien común, sin buscar una recompensa financiera (Delfgaauw y Dur 2010).

\subsection{Gestión del Talento en la literatura}

Durante la última década, la importancia de la Gestión de los Recursos Humanos como un elemento clave en la gestión de una empresa se ha consolidado y la Gestión del Talento ha surgido como la mejor estrategia para gestionarlos aplicada a diferentes campos.

El principal problema al abordar la Gestión del Talento, académicos y profesionales por igual, es la definición de "Talento" y "Gestión de Talento". Hay poco consenso sobre ambos conceptos, pero las características generales coinciden, a pesar de que muchos otros dependen de elementos como los culturales (Zhang y Bright, 2012; Li et al., 2018). Finalmente se ha optado por la definición de Talento de Tansley basada en el origen etimológico de la misma: "una habilidad natural especial o aptitudes y facultades de varios tipos (órdenes mentales de un orden superior, poder o habilidades mentales) (Tansley, 2011:267). Y, para definir "Gestión del talento", muchas definiciones pueden encajar en el contexto occidental, pero la de Nijs y sus colaboradores resulta una de las más completas: "el talento se refiere a las habilidades innatas desarrolladas sistemáticamente en las personas que se despliegan en actividades que les gustan, que encuentran importantes y en las que desean invertir energía. Permite que los individuos se desempeñen de manera excelente en uno o más dominios del funcionamiento humano, interviniendo para que tengan un mejor desempeño que otros individuos de la misma edad o experiencia, o que se desempeñen de manera permanente en su mejor nivel personal" (Nijs et al., 2014:7).

Establecidos los dos conceptos principales, se detecta en la revisión general de la literatura una producción científica que ha aumentado, exponencialmente, para explorar su incidencia sobre la práctica. En este contexto, principalmente se busca conceptualizar un enfoque sistemático acerca del alcance de la gestión del talento en "grupos de talentos" de corporaciones multinacionales (Jooss et al., 2019), es decir, estructuras empresariales que operan y compiten globalmente con sistemas de gestión que proporciona un gran potencial para enriquecer directa o indirectamente la capacidad organizacional, identificando la visión macro del talento como eje principal (King y Vaiman, 2019). 
No obstante, a nivel micro, se analiza con resultados positivos y significativos, múltiples mejoras en el mundo empresarial gracias a la gestión del talento; sirva de ejemplo, su influencia sobre la satisfacción laboral y el compromiso organizacional (Alparslan y Saner, 2019), su relación con el género (Makarem et al., 2019) o cómo reinventarse en la "nueva normalidad" poniendo de relieve la importancia en la innovación de modelos de negocios (Loon et al., 2020). Por tanto, el talento se convierte en una herramienta de gestión muy versátil y adaptable a las organizaciones.

Para el propósito de este trabajo, las búsquedas de literatura han sido limitadas a Turismo y/o Hotelería, obteniendo pocos resultados. Apenas 33 trabajos accesibles (marzo de 2019) cuyas conclusiones y preocupaciones van en la misma dirección: altos índices de rotación y retención (Barron, 2008; Deery, 2008; Hausknecht et al., 2009; Alferaih et al., 2018; Li et al., 2018) y conciliación laboral (Deery, 2008; Deery y Jago, 2015). Además, cabe mencionar que muchos de ellos se limitan a un contexto territorial específico tales como la India (Bharwani y Talib, 2017; Jauhari y Sanjeev, 2012), Arabia Saudí (Alferaih et al., 2018; Alferaih, 2017), Escocia (Maxwell y MacLean, 2008; Bratton y Waton, 2018) o China (Li et al., 2018; Ma et al., 2019), por lo que se recomienda precaución en la extrapolación de datos y conclusiones.

Además, dado que el objetivo de este estudio es analizar la Administración Pública en el área de Turismo, se aplicaron limitaciones al sector público, sin encontrar literatura específica alguna.

\subsection{Teoría del Valor}

Esta teoría fue desarrollada por Sparrow y Makram (2015) como resultado de una compilación analítica y simplificada de las principales filosofías sobre el tema. Consta de varias fases que pueden aplicarse en cualquier campo, incluido el sector público, por lo que se ha adoptado como base teórica de este trabajo. En primer lugar, identificaron cuatro enfoques o filosofías diferentes de la Gestión del Talento que actúan como una clasificación de la literatura anterior en la materia y subyacen en un marco asentado en el concepto de "valor":

“1) Enfoque de personas: la Gestión del Talento como una categorización de personas;

2) Enfoque de prácticas: la Gestión del Talento como la presencia de prácticas clave de gestión de recursos humanos;

3) Enfoque de posición: la Gestión del Talento como la identificación de posiciones oscilantes en el contexto del diseño, control y coordinación de la organización;

4) Enfoque de grupos estratégicos: la Gestión del Talento como grupos de talentos internos y planificación de la sucesión” (Sparrow y Makram, 2015: 250-251).

En segundo lugar, establecieron cuatro fases (Figura 1) como un solo mecanismo para construir y distribuir una capacidad más amplia en torno a la organización: 


\subsubsection{Creación de valor}

Incluye todos los procesos destinados a atraer, adquirir y reunir recursos de talento (candidatos con talento) que pueden ser de algún valor para la organización. Dichas personas son valiosas por sí mismas en el ámbito laboral debido a sus propias habilidades y conocimientos (iniciativa, autonomía, competencias profesionales específicas o desempeño creativo) pero se espera que la empresa experimente un aumento de dicho valor, por lo que se lograría una mayor ventaja competitiva.

Por otro lado, la organización ha de ser atractiva para conquistar valor al generar una buena reputación y garantías laborales, independientemente si busca el valor del empleado en tiempo y esfuerzo, en resultados de su creatividad o ponderando los costes y beneficios.

\subsubsection{Captura de valor}

Es el proceso por el cual las organizaciones agrupan a los empleados talentosos y los recursos disponibles con el objetivo de crear una relación de dependencia entre ambos, por lo que el poder de negociación de los empleados se reduce considerablemente. Algunos autores difieren en cómo se debe crear dicha relación de dependencia y cómo afecta a la creación de valor (Brandenburger y Nalebuff, 1995; Chatain y Zemsky, 2011; Stirling, 2011). Pero todos están de acuerdo en que atrapar valor es esencial para las organizaciones, por lo que la captura de empleados talentosos, sobre todo en la industria del turismo, es necesaria para tener una ventaja sobre sus competidores. Esta etapa podría aplicarse a los empleados del sector público si se introduce un enfoque estratégico en el modelo de selección junto a las condiciones ofrecidas por la organización pública, incluidos el salario, el entorno laboral y la potencial carrera profesional.

\subsubsection{Apalancamiento de valor}

En esta etapa, una vez que los empleados talentosos son capturados, las organizaciones desarrollan e implementan su talento para agregar valor a la calidad de los productos y/o servicios. No se trata de crear valor sino de agregarlo a los productos y servicios ya existentes para aumentar su valor. Aunque a primera vista parece bastante similar a la creación de valor, la diferencia es que, en esta etapa, el valor no es solo el resultado de la creatividad, sino su aplicación a algo que ya existe. En este sentido, el diseño estratégico de los puestos de trabajo potenciaría el valor del empleado y la calidad del servicio que presta.

\subsubsection{Protección de valor}

La retención de empleados talentosos evitaría la marcha a otras empresas a pesar de que, en ciertos aspectos, les ofrezcan mejores condiciones que su organización actual. Parece lógico pensar, si otra empresa ofrece mejores condiciones generales, él/ella se marcharía si tuviera la oportunidad. Sin embargo, es responsabilidad de cada organización proteger sus activos más valiosos al proporcionarles condiciones imbatibles o mejorar las 
actuales. Tanto en el ámbito público como en el privado, la promoción interna se convertiría en un gran aliado de la protección del valor junto a la capacitación especializada orientada al desarrollo de nuevos productos/servicios, entendiendo en el ámbito público la protección como fidelización de sus efectivos para mantener un servicio público de calidad y evitar el presentismo.

\section{Figura 1 \\ FASES DE LA TEORÍA DEL VALOR}

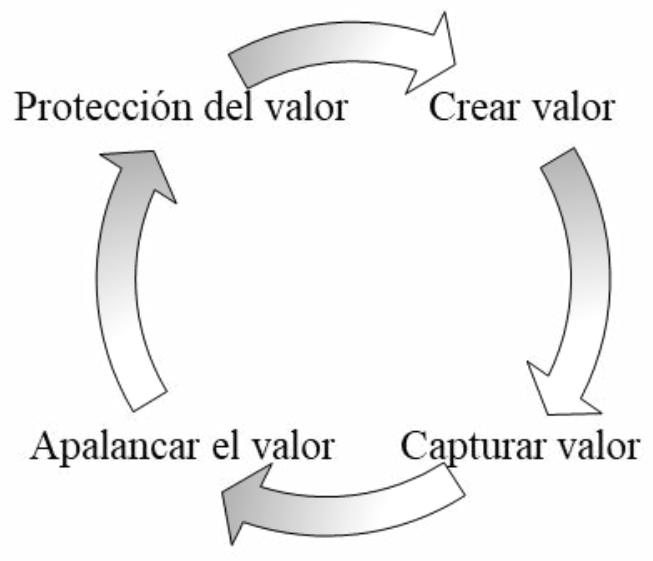

Fuente: Sparrow y Makram (2015)

Como señalamos en la revisión de la literatura, el consenso académico sobre cómo definir el talento aún genera discusión. Sin embargo, la práctica de su gestión parece haber llegado a un acuerdo, en gran medida unánime, acerca de las etapas que lo conforma: crear, capturar, apalancar y proteger. A esta perspectiva del valor se suma la dependencia del contexto, cuyo propósito es crear una ventaja competitiva sostenible mediante la reconfiguración de los recursos en los entornos cambiantes y evolutivos de las organizaciones (Harsch y Festing, 2020).

Siguiendo la línea de esta teoría, podemos observar en variados trabajos su aplicación en "casos de estudio" que analizan, por ejemplo, cómo influye en las fases del proceso de gestión del talento el tamaño de las empresas en Eslovaquia, observando una mayor importancia en la creación y captura de talento para grandes empresas mientras que para las PYMES resulta más ventajosa su retención (Savov et al., 2020). Igualmente, se aplica en la práctica de PYMES en Polonia, desarrollando un enfoque que presta especial atención a sus características y contexto ambiental (factores externos e internos) y cultural (Pauli y Pocztowski, 2019).

Se debe agregar, un caso sobre sector público, donde se analiza la gestión estratégica de los recursos humanos de las Fuerzas Armadas en Portugal como factor determinante de protección de talento. A este colectivo, las inversiones en términos de formación académica, técnica y operativa han contribuido a incrementar sus habilidades y destrezas en 
su carrera profesional, sin embargo, las diferentes aspiraciones profesionales vinculadas a limitaciones organizativas, distintas asignaciones económicas y los variados contextos hacen de la gestión del talento una herramienta aconsejable para la retención de valor entre sus filas a pesar de la rigidez estructural (Rosado et al., 2019).

Siguiendo con el mismo sector, pero en materia de turismo, proteger el valor parece ser bastante complicado ya que los superiores directos no tienen poder de negociación. Las características y condiciones de cada trabajo vienen preestablecidas de modo que hay poco margen para negociar en contra de la oferta de otra organización, por tanto, cuando se trata de retener a empleados valiosos les resulta muy difícil. La única ventaja de este sistema, amparada en la Oferta Pública de Empleo, recae sobre los empleados públicos. Por un lado, aquellos que obtienen un puesto de trabajo como funcionarios de carrera reducen al mínimo el riesgo de ser despedidos y mantienen turnos estables, no obstante, esta modalidad es bastante reducida en esta área. Y, por otro lado, los que conforman la gran mayoría de sus efectivos, personal laboral fijo, con escaso riesgo de despido, pero con turnos rotatorios y/o personal laboral temporal con contratos que, en las actuales condiciones del mercado turístico, se han visto reducidos drásticamente.

En consecuencia, podría concluirse que las organizaciones del sector público, al igual que las empresas privadas, pueden crear, capturar y aprovechar el valor a través de las prácticas de Gestión del Talento. Sin embargo, difieren de las empresas del sector privado en la protección del valor, ya que son escasas las herramientas para retener a sus empleados talentosos.

\subsection{Sector público y formas contractuales en el turismo}

Últimamente, en España, conseguir un trabajo como funcionario público se ha convertido en una opción interesante para muchas personas que buscan un trabajo estable. Esa decisión no solo la toman quienes ya han trabajado en el sector privado, sino que se ha convertido en un deseo de muchos estudiantes. Los últimos estudios encontrados en el contexto de España, ambos realizados en 2017, muestran que entre el 26,79\% (ESIC: Marketing \& Business School \& Praxis MMT Corporation, 2017) y el 31,8\% (GAD3, Educa 20.20 y Fundación AXA, 2017) de los estudiantes de nivel preuniversitario prefieren trabajar dentro de la Administración Pública. Eso es impactante ya que solo Italia, considerando otros países mediterráneos, tiene cifras similares con un 24,18\% de sus estudiantes dispuestos a trabajar para el sector público (ESIC: Marketing \& Business School \& Praxis MMT Corporation, 2017 ) mientras que Portugal $(5,98 \%)$ o Grecia $(14,05 \%)$ se encuentran lejos de estas cifras.

Sin embargo, obtener un empleo como funcionario público u otro tipo de contratos en este sector no es fácil. La gran mayoría se obtienen a través de concurrencia competitiva, por lo que, debido a la gran demanda de empleo en la Administración Pública, la probabilidad de obtener uno de ellos es muy baja. Por ejemplo, durante el primer trimestre de 2018, se ofertaron a nivel nacional 4.725 empleos para el conjunto público (sanidad, educación, turismo, fuerzas y cuerpos de seguridad del Estado, etc.), pero más de 82.000 personas se inscribieron para tomar parte en las pruebas selectivas (Ricou, 2018). Es decir, menos del 5\% de los candidatos finalmente obtendrían un puesto de trabajo. Actualmente, estos exámenes se consideran la mejor manera de seleccionar a esa élite de candidatos, basados principal- 
mente, en ejercicios memorísticos en lugar de la evaluación de habilidades y destrezas. De ello, se derivan problemas en aquellos empleos en los que se trabaja directamente con el público o cuando se requiere un trabajo creativo como es el caso del sector turístico.

Por lo tanto, se considera esencial, analizar los medios de acceso a los empleos del sector público y sus tipos de nombramientos/contratos [Tabla 1] con el propósito de evaluar adecuadamente los empleos en el área de Turismo considerando los posibles problemas asociados con las habilidades de los empleados.

\section{Tabla 1 \\ FORMAS CONTRACTUALES DEL SECTOR PÚBLICO}

\begin{tabular}{ll}
\hline & - Empleados con una relación permanente con la Administración pública. \\
Funcionario & - Realizan tareas que están reservadas al Estado. \\
de carrera & Separación del servicio por causas tasadas en EBEP. \\
& - Promoción en diferentes grupos y subgrupos profesionales: Grupo A [A1 y A2], \\
& Grupo B y Grupo C [C1 y C2].
\end{tabular}

Funcionario

- Empleados que aún no son funcionarios de carrera pero que, en ausencia de interino funcionario, realizan las mismas tareas por sustitución, vacante, ejecución de programas de carácter temporal o acumulación de tareas.

\begin{tabular}{cc}
\hline & - Empleados públicos con una relación contractual fija, indefinida y realizan \\
& tareas específicas no reservadas a los funcionarios de carrera. \\
Personal & - Se regirá por la legislación laboral y demás normas convencionalmente \\
laboral fijo & aplicables. \\
& La probabilidad de ser despedido es algo mayor que un funcionario de carrera. \\
\hline Personal & - Empleados con una relación temporal con la Administración Pública. \\
laboral & - Son contratados para tareas específicas por la autoridad competente según los \\
temporal & términos que marca la ley. \\
\hline Personal & En virtud de nombramiento y con carácter no permanente, sólo realiza funciones \\
eventual & $\begin{array}{l}\text { expresamente calificadas como de confianza o asesoramiento especial, siendo } \\
\text { retribuido con cargo a los créditos presupuestarios consignados para este fin. }\end{array}$ \\
& $\begin{array}{l}\text { Sus condiciones no son reguladas por convenio colectivo y en lo que sea } \\
\text { adecuado a la naturaleza de su condición, el régimen general de los funcionarios } \\
\text { de carrera }\end{array}$
\end{tabular}

Fuente: Elaboración propia a partir de EBEP

Según el Estatuto Básico del Empleado Público (Boletín Oficial del Estado, 2015) -EBEP en adelante - ley que regula el empleo en este sector, todos los ciudadanos pueden acceder a un puesto del sector público. Asimismo, dicho acceso está protegido por el artículo 103 de la Constitución española (CE, 1978), que consagra los derechos 
constitucionales de equidad, mérito y capacidad. Además, los artículos 56 a 59 del EBEP (Boletín Oficial del Estado, 2015) regulan los requisitos específicos, como ser español, poder desarrollar las tareas involucradas en el trabajo, incluso el tratamiento especial de las personas con discapacidad, la edad mínima de ingreso y la edad máxima previa a la jubilación, entre otros.

Además, el artículo 55 del EBEP (Boletín Oficial del Estado, 2015) regula las ofertas de empleo público que deben cumplir los requisitos de publicidad de la oferta y sus condiciones, transparencia, imparcialidad y profesionalidad de los miembros de los órganos de selección, así como la independencia y la discreción técnica de los mismos, la relación entre los contenidos de las pruebas y las tareas a realizar. En adición, el artículo 61 de la EBEP (Boletín Oficial del Estado, 2015) incluye los métodos para seleccionar empleados. Son similares y dependen de la oferta de trabajo que se lleve a cabo, es decir, tanto para los funcionarios públicos como para el personal laboral, la forma utilizada puede ser un examen público, concurso o ambos. Finalmente, el Título II de la EBEP (Boletín Oficial del Estado, 2015) establece el tipo de formas contractuales posibles dentro de la Administración Pública.

\section{EL TURISMO EN LA ADMINISTRACIÓN PÚBLICA EN ANDALUCÍA: ORGANIZACIÓN Y EMPLEADOS}

El sector público es bien conocido por todos como un enorme sistema burocrático, con muchas oficinas en todo el país, cualquiera que sea el país, algo parecido a un enorme árbol con cientos de ramas. En este estudio, como se indica en la introducción, la unidad política y territorial elegida no es España sino Andalucía, por lo que puede hacer pensar que ha resultado un análisis más fácil de manejar. Nada podría estar más lejos de esa idea porque Andalucía es una extensa región y, como el resto de las regiones en España, hasta tres Administraciones Públicas tienen competencias en temas de Turismo, además de cumplir con las directrices de la Administración General del Estado. Teniendo en cuenta esta situación, no hace falta decir que el análisis realizado es más complejo de lo que pudiera parecer.

De acuerdo con el artículo 148 de la Constitución Española (CE, 1978), el turismo puede ser, y es, competencia de las Comunidades Autónomas. Literalmente, dice: "Promoción y planificación del turismo en su territorio”. En consecuencia, Andalucía lo reglamentó a través de una ley (Ley de Turismo de Andalucía) que incluía una regulación exhaustiva de todas las actividades relacionadas con el turismo, reiterando tal poder otorgado por la Constitución en el Artículo 3 (LTA, 2011).

Además, el artículo 7 y 27 de la Ley 7/1985, que regula la base de los regímenes locales (Boletín Oficial del Estado, 1985), establece que el Estado y las Comunidades Autónomas pueden delegar en las Entidades Locales sus competencias. Como resultado, los Municipios - Artículo 25 de la Ley 7/1985 (Boletín Oficial del Estado, 1985) - y Diputaciones Provinciales - Artículo 36 de la Ley 7/1985 (Boletín Oficial del Estado, 1985) y los Artículos 11 a 15 de la Ley 5/2010 (Boletín Oficial del Estado, 2010) - de Andalucía disfrutan de competencias en turismo. 


\section{Figura 2 \\ ÓRGANOS DE LA JUNTA DE ANDALUCÍA RELACIONADOS CON EL TURISMO}

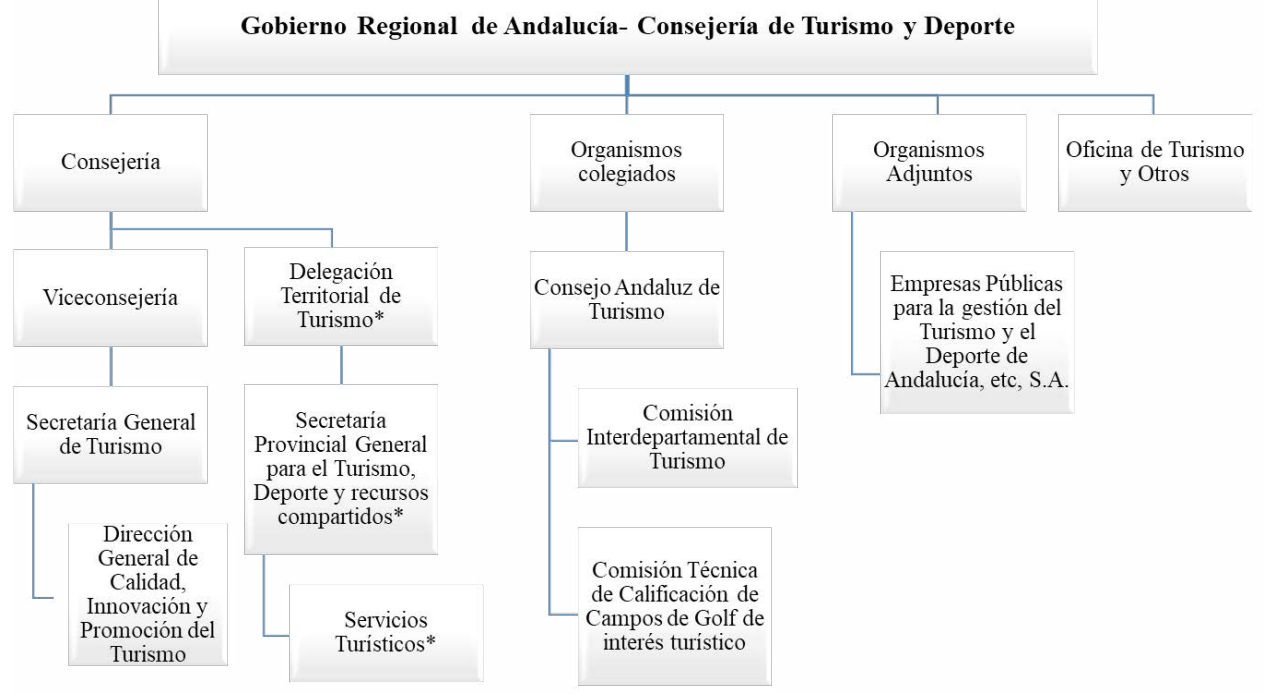

Fuente: Elaboración propia.

Como consecuencia de la dispersión de las competencias en turismo en tres administraciones públicas (Figura 2), se crearon muchas organizaciones públicas a lo largo de los años. Además, otras competencias relacionadas con la esfera del turismo, pero no incluidas en las competencias de promoción y planificación, como competencias costeras, influyen indirectamente en la actividad turística. Las organizaciones públicas relacionadas con estas últimas competencias no se consideraron al realizar la investigación, ya que no están relacionadas directamente.

Una vez que se conocen todas las administraciones públicas con competencias en turismo en Andalucía, se descubrieron un par de contratiempos. Por un lado, identificar cuántas oficinas, dónde están y a qué Administración están subordinadas resulta ser bastante difícil ya que no hay un registro oficial que las inventaríe. Por otro lado, descubrir cuántos empleados trabajan en cada uno de ellos para poder realizar una encuesta, que se desarrolla en la siguiente sección, se hizo imposible debido a una marcada falta de transparencia y la fusión de varias competencias en la misma organización, por lo que sus directorios y servicios se unen sin importar, por ejemplo, que el personal a cargo de Deportes no está involucrado en temas de turismo. Para tratar de aproximar el número de empleados del sector público que trabajan en el ámbito del turismo en Andalucía, se muestran y agrupan el organigrama de las Administraciones Públicas actuales relacionadas con el sector del Turismo de acuerdo con la Administración principal a la que pertenecen. Cabe destacar que éste muestra solo las oficinas y organizaciones directamente relacionadas con el turismo, evitando otras que brindan servicios generales, como la Secretaría 
Técnica General, o que realicen actividades en otras áreas, como la Secretaría General de Deportes, con el fin de enfocarnos en el área de Turismo exclusivamente.

Además, se necesitan algunas indicaciones adicionales para una mejor comprensión. En primer lugar, algunas oficinas están marcadas con un asterisco, lo que significa que hay una de ellas en cada provincia [Tabla 2]. En segundo lugar, la sucursal que contiene los organismos adjuntos solo muestra la Empresa Pública para la Gestión del Turismo y el Deporte en Andalucía, S.A., que actualmente es la única relacionada con el turismo perteneciente a la Secretaría de Turismo y Deporte de la Región. Hace algún tiempo, Cetursa, S.A. y Promonevada, S.A. pertenecían a la Consejería de Turismo y Deporte, pero hoy en día, ambos pertenecen a la Consejería de Hacienda, Industria y Energía. Además, Promonevada, S.A. se encuentra actualmente en proceso de liquidación [Tabla 3].

\section{Tabla 2}

\section{ORGANISMOS E INSTITUCIONES RELACIONADAS CON EL TURISMO}

\begin{tabular}{ll}
\hline Diputaciones Provinciales & $\begin{array}{l}\text { Diputaciones* } \\
\text { Oficinas de Turismo y Otros }\end{array}$ \\
\hline Municipios & $\begin{array}{l}\text { Ayuntamientos } \\
\text { Oficinas de Turismo y Otros }\end{array}$ \\
\hline Otros & Cámaras de Comercio* \\
\hline
\end{tabular}

Fuente: Elaboración propia.

Como se indicó anteriormente, no ha sido fácil recopilar la información contenida en la Tabla 3, en ella se ha estimado el número de empleados públicos en turismo en Andalucía pero con ciertas limitaciones, debido a las siguientes razones:

1. A pesar de que se encuentran las Instituciones administrativas y su dirección oficial, no se encuentran datos sobre el número de empleados ni el área específica de trabajo para la Secretaría General Provincial de Turismo, Deporte y Recursos Compartidos y en el Servicio de Turismo. Ambas pertenecen a la Delegación Territorial de Turismo de la Consejería de Turismo y Deporte, por lo que hay uno en cada provincia.

2. En cuanto a los órganos colegiados, el Consejo Andaluz de Turismo, la Comisión Interdepartamental de Turismo y la Comisión Técnica para la Clasificación de los Campos de Golf de Interés Turístico parecen no tener empleados contratados. Constan representantes considerados como altos cargos dentro de las oficinas de la Consejería, de acuerdo con sus leyes reguladoras: los artículos 7 y 8 de la Ley 13/2011 (Boletín Oficial del Estado, 2011) en los dos primeros casos y el artículo 30 del Decreto 43/2008 - que regula las condiciones de establecimiento y funcionamiento de los campos de golf en Andalucía (Boletín Oficial de la Junta de Andalucía, 2008) - para este último. Ninguna evidencia mostró empleo adicional para estos cuerpos.

3. En el caso de las Diputaciones Provinciales en Cádiz, Córdoba y Granada tienen disponible su cronograma de recursos humanos, pero no está clara la información que se muestra. La Diputación Provincial de Cádiz no divide su personal en áreas, mientras que las de Córdoba y Granada lo hacen, pero no muestran ningún área de 
Turismo. Finalmente, las Cámaras de Comercio de Almería y Huelva no muestran cuántas personas trabajan para ellas, pero sí muestran cuánto gastan en salarios. Dependiendo de cuántos puestos ejecutivos tengan, el número de empleados puede variar, pero se puede inferir un rango de empleados de 23 a 37, con respecto a otros gastos de las Cámaras de Comercio - Granada y Málaga, respectivamente - en salarios. Sin embargo, tales números no se consideran debido a la variabilidad del rango. La Cámara de Comercio de Jaén existía, pero actualmente está esperando un proceso de liquidación debido a una importante deuda.

Con respecto a los datos contenidos en la Tabla 3, también se necesitan algunas explicaciones, detalladas en la parte metodológica de este trabajo, debido a que en algunos casos el número de empleados es explícito y exacto, pero en otros muchos no.

\section{Tabla 3 \\ CUANTIFICACIÓN DE LOS EMPLEADOS PÚBLICOS EN EL ÁREA DE TURISMO DE ANDALUCÍA}

\begin{tabular}{|c|c|c|c|c|}
\hline Consejería & $\begin{array}{c}\text { Secretaría General } \\
\text { Provincial de Turismo, } \\
\text { Deporte y Recursos } \\
\text { Compartidos }\end{array}$ & $\begin{array}{c}\text { Consejo Andaluz de } \\
\text { Turismo }\end{array}$ & $\begin{array}{c}\text { Empresas } \\
\text { Públicas para } \\
\text { la gestión del } \\
\text { Turismo y } \\
\text { Deporte, S.A. }\end{array}$ & \\
\hline 12 & Sin datos publicados & Sin datos publicados & 180 & \\
\hline Viceconsejería & Servicios de Turismo & \begin{tabular}{|c|} 
Comisión \\
Interdepartamental de \\
Turismo
\end{tabular} & & \\
\hline 47 & Sin datos publicados & \begin{tabular}{|l|} 
Sin datos publicados \\
\end{tabular} & & \\
\hline $\begin{array}{c}\text { Secretaría } \\
\text { General de } \\
\text { Turismo }\end{array}$ & $\begin{array}{c}\text { Dirección General para } \\
\text { la Calidad, Innovación } \\
\text { y la Promoción del } \\
\text { Turismo } \\
\end{array}$ & \begin{tabular}{|c|} 
Comisión Técnica \\
para la Calificación de \\
Campos de Golf de \\
Interés Turístico \\
\end{tabular} & & \\
\hline \multirow[t]{2}{*}{92} & 44 & Sin datos publicados & & \\
\hline & $\begin{array}{c}\text { Delegaciones } \\
\text { Territoriales de } \\
\text { Turismo } \\
\end{array}$ & $\begin{array}{c}\text { Diputaciones } \\
\text { provinciales }\end{array}$ & $\begin{array}{c}\text { Cámaras de } \\
\text { Comercio }\end{array}$ & $\begin{array}{c}\text { Oficinas de } \\
\text { Turismo/Otros }\end{array}$ \\
\hline Almería & 121 & 27 & $\begin{array}{c}\text { Sin datos } \\
\text { publicados }\end{array}$ & 85 \\
\hline Cádiz & 146 & Sin datos publicados & 11 & 123 \\
\hline Córdoba & 153 & Sin datos publicados & 34 & 110 \\
\hline Granada & 154 & Sin datos publicados & 23 & 75 \\
\hline Huelva & 140 & 16 & $\begin{array}{c}\text { Sin datos } \\
\text { publicados }\end{array}$ & 68 \\
\hline Jaén & 140 & 25 & No existe & 75 \\
\hline Málaga & 159 & 72 & 37 & 145 \\
\hline Sevilla & 158 & 132 & 69 & 90 \\
\hline \multirow[t]{2}{*}{ Total } & 1.171 & 272 & 174 & 771 \\
\hline & $\begin{array}{c}\text { Gobierno Regional de } \\
\text { Andalucía }\end{array}$ & Consejos Provinciales & Municipios & $\begin{array}{c}\text { Otros } \\
\text { O.T. y C.C. }\end{array}$ \\
\hline Total & 1.546 & 272 & 578 & 945 \\
\hline 3.341 & & & & \\
\hline
\end{tabular}

Fuente: Elaboración propia. 
Para terminar, estimamos que un total de aproximadamente 3.341 empleados trabajan entre todas las Administraciones Públicas en el ámbito del turismo en Andalucía. Por lo tanto, todos ellos se consideran empleados del sector público, pero cabe destacar que, después de revisar las Relaciones de Puestos de Trabajo, se observa que la mayoría de ellos no son funcionarios de carrera sino contratados como personal laboral fijo o, a veces, laboral temporal.

\section{METODOLOGÍA}

En este epígrafe se realiza, por un lado, la estimación del número de empleados públicos de turismo en Andalucía y por otro, la evaluación sobre qué piensan los empleados del sector público sobre la Gestión del Talento y la aplicabilidad a sus trabajos.

\subsection{Estimación número de empleados públicos en turismo de Andalucía}

Las dificultades en el cálculo estimatorio del número de trabajadores en el área de turismo, ha determinado la inclusión de varias aclaraciones a partir de las evidencias de los datos publicados:

1. La Relación de Puestos de Trabajo (RPT) de la Consejería, la Viceconsejería, la Secretaría General de Turismo y la Dirección General de Calidad, Innovación y Promoción del turismo son exactos, ya que solo cuentan con su propio personal. Por el contrario, las Delegaciones Territoriales se denominan Delegaciones Territoriales de Turismo, Regeneración, Justicia y Administraciones Locales, por lo que incluyen en el mismo registro a todos los empleados sin diferenciar sus áreas. Para evitar cometer errores, solo se restaron aquellos empleados que parecen evidentes que no trabajan en el área de turismo, como conductores, personal de limpieza o personal de mantenimiento. Este es también el caso de la Empresa Pública para la Gestión del Turismo y el Deporte en Andalucía, S.A. En consecuencia, cuando se trata de dichos datos, se debe considerar que los números que se muestran son probablemente menores.

2. En el caso de los datos de las Diputaciones Provinciales y las Cámaras de Comercio, estos números son exactos y se extraen directamente de sus respectivas RPT.

3. Con respecto a los municipios, para lograr una aproximación del número de empleados que trabajan en ellos en el área de turismo, se necesita una inferencia. Dicha inferencia se basa en la experiencia de los autores de este trabajo al realizar la encuesta que se muestra en la siguiente sección, así como en el número de lugares de alojamiento, que se considera comúnmente como un indicador del desarrollo turístico. En este sentido, todos los municipios andaluces fueron ordenados por los lugares de alojamiento de sus hoteles, utilizando la base de datos SIMA (Instituto de Estadística y Cartografía de Andalucía, 2017), que mostró que 504 de 786 municipios tienen lugares de alojamiento, 47 de los cuales tienen más de 1.000 y 9 de ellos con más de 10.000. Luego, se llevó a cabo una búsqueda de cuántos empleados trabajan en los municipios más grandes, generalmente, entre 5 a 10 , y los que se encuentran en los municipios más pequeños, generalmente, 1 o ninguno. En consecuencia, se calculó un promedio considerando una media 
de 5 empleados para esas oficinas en municipios con más de 1.000 alojamiento y 0,75 en aquellos con menos de 1.000 , resultando en un promedio de 1,15 , que fue el multiplicador aplicado al número de municipios.

Finalmente, con respecto a las Oficinas de Turismo y Otros, que incluyen Centros de Visitantes, Puntos de Información de todas las Administraciones: Delegaciones Territoriales de Turismo, Diputaciones Provinciales y Municipios. Se consideraron juntos, ya que la información de cuántos de ellos existen se proporciona de esa manera mediante la herramienta de búsqueda utilizada (Empresa Pública para la Gestión del Turismo y el Deporte de Andalucía, S.A., 2019). Sin embargo, la información recuperada es el número de oficinas -308 - pero no el número de empleados, por lo que, como anteriormente, era necesaria una inferencia siguiendo el mismo método. En este caso, dado que el cronograma de personal de muchas oficinas de turismo es casi imposible de encontrar, la inferencia se basa exclusivamente en la experiencia de los autores al realizar la encuesta mencionada anteriormente. Por lo tanto, se consideró que los municipios grandes tienen alrededor de 7 personas trabajando en los más grandes y alrededor de 2 o 3 empleados en los más pequeños, y los municipios pequeños tienen, en general, solo 1 empleado. En consecuencia, el multiplicador utilizado es 2,5 aplicado al número de oficinas.

\subsection{Cuestionario y validación}

En primer lugar, se ha diseñado, atendiendo al objetivo principal de la investigación, un cuestionario exploratorio (ver Anexo) orientado a conocer las percepciones de los empleados públicos en línea con el marco teórico desarrollado, pues se pretende abrir un campo de trabajo poco analizado hasta el momento. En segundo lugar, se han planteado preguntas y cuestiones cortas, con un lenguaje sencillo, evitando redundancias y excesivos campos abiertos. Asimismo, su extensión ha sido reducida (21 preguntas escala de Likert de 1 a 5) con la finalidad de que su breve duración (5 minutos) no provocara excesivo rechazo entre la población objeto de estudio.

La encuesta se realizó a través de un muestreo aleatorio estratificado del 12 al 21 de marzo de 2019 y se envió telemáticamente el cuestionario piloto a un grupo reducido de 500 empleados públicos, obteniendo 116 respuestas de las que se seleccionaron 100 válidas sobre un total estimado de 3.300 sujetos, según nuestras estimaciones en el sector [Tabla 3], mediante envíos a las delegaciones territoriales de Turismo de la Junta de Andalucía, a las oficinas de turismo, a los Ayuntamientos de costa y de interior y a las Diputaciones provinciales del conjunto de la Comunidad Autónoma.

El número de sujetos de la muestra podría parecer débil con respecto al necesario, 345 cuestionarios con un nivel de confianza del 95\% y un margen de error del 5\%, sobre el total estimado para la población de estudio. No obstante, el objetivo ha sido realizar un primer acercamiento para conocer si hemos tenido la suficiente empatía para colocarnos en el lugar del entrevistado y confirmar la fiabilidad y validez del cuestionario. Para ello, según las recomendaciones de Mondéjar (2016) es conveniente escoger una pequeña muestra, normalmente inferior a 100 individuos, con características similares a la población de estudio. 
Tabla 4

ANÁLISIS DE FIABILIDAD COMPUESTA DEL CUESTIONARIO

\begin{tabular}{|c|c|c|}
\hline Estadísticos de fiabilidad & $\begin{array}{c}\text { Correlación } \\
\text { total }\end{array}$ & $\begin{array}{c}\text { Alfa de } \\
\text { Cronbach }\end{array}$ \\
\hline $\begin{array}{l}\text { 9. ¿Considera que tiene autonomía y libertad en el desarrollo de las } \\
\text { tareas? }\end{array}$ & ,020 & $\mathbf{8 9 5}$ \\
\hline 10. ¿Se considera competente para desarrollar su trabajo? & ,635 & ,875 \\
\hline $\begin{array}{l}\text { 11. ¿Su trabajo le da la oportunidad de mejorar sus habilidades } \\
\text { profesionales? }\end{array}$ & ,466 & ,883 \\
\hline 12. ¿Su trabajo requiere tener iniciativa y un desempeño creativo? & ,646 & ,874 \\
\hline $\begin{array}{l}\text { 13. ¿Considera que la institución le oferta una buena formación } \\
\text { acorde con su trabajo? }\end{array}$ & ,710 & ,870 \\
\hline 14. ¿Tiene oportunidad de crecer personalmente en el trabajo? & ,799 & ,867 \\
\hline 15. En general, ¿se encuentra satisfecho con su trabajo? & ,597 & ,877 \\
\hline $\begin{array}{l}\text { 16. Considero acertado el modelo de selección de personal en la } \\
\text { Oferta de Empleo Público }\end{array}$ & ,764 & ,867 \\
\hline 17. Considero adecuado el modelo de promoción interna & ,656 & ,873 \\
\hline 18. Creo ajustado el diseño de los puestos de trabajo & ,163 & 9896 \\
\hline $\begin{array}{l}\text { 19. Opino que es necesaria la evaluación del desempeño en los } \\
\text { términos que establece el Estatuto Básico del Empleado Público } \\
\text { (EBEP) }\end{array}$ & ,203 & ,893 \\
\hline 20. Considero importante dar un enfoque estratégico a mi trabajo & ,816 & ,866 \\
\hline 21. Me siento satisfecho/a en el trabajo & ,816 & ,866 \\
\hline
\end{tabular}

Fuente: Elaboración propia.

Además de la información relacionada estrictamente con los problemas de Gestión del Talento [Tabla 4], también se recopiló información socio-laboral con el objetivo de lograr una mejor comprensión del conjunto de empleados públicos.

Para la validación del cuestionario fue necesario evaluar la consistencia y la coherencia interna de cada uno de sus ítems, por lo que se realizó un estudio psicométrico de fiabilidad utilizando el software SPSS.

En este sentido, para determinar la validez y fiabilidad se utilizaron tres procesos complementarios: el coeficiente alfa de Cronbach, un análisis de fiabilidad compuesta y la varianza 
extraída media (AVE). Según Nunnally y Bernstein (1994), el coeficiente alfa de Cronbach, como valor de fiabilidad aceptable para evaluar las correlaciones entre las variables, debe ser superior a 0,8 . En el caso de este estudio, el resultado es 0,886 , por lo que se demuestra que es fiable. En segundo lugar, el análisis de fiabilidad compuesta nos indica aquellas variables que aportan o no una mayor fiabilidad al cuestionario. Esta técnica será útil para eliminar aquellas variables que no contribuyen a mejorar la encuesta, evitando problemas posteriores en el procesamiento e interpretación de datos. Tomando la correlación total de los elementos corregidos se muestra que solo tres variables presentan un valor inferior a 0,3 [Tabla 4], que es el valor mínimo de referencia (Werts et al., 1974), pero la correlación entre las demás variables confirma que el cuestionario es internamente coherente.

Finalmente, para analizar la validez convergente, se utiliza la varianza extraída media (AVE), que resulta en 0,57868023. Fornell y Larcker (1981) sugieren que 0,5 es el valor mínimo aceptable, por lo que, en consecuencia, el ajuste de las variables de este estudio es significativo y está altamente correlacionado.

\subsection{Resultados}

En primer lugar, para tener una visión general del conjunto de empleados, se muestra el arquetipo de empleado del sector público utilizando los datos gráficos socio-laborales recopilados. Es una mujer (67\%) mayor de 45 años $(45,9 \%)$, graduada universitaria o más $(79,8 \%)$ que ha estado trabajando en el área de Turismo de 10 a 20 años $(32,1 \%)$ como personal permanente o temporal, no como funcionaria de carrera $(76,2 \%)$ y que no ha podido promocionar $(67,3 \%)$. Es notable la edad que tiene el personal a pesar de que, en general, son más jóvenes que a nivel estatal, cuyo porcentaje de empleados mayores de 45 años es del 58,47\% (Instituto Nacional de Estadística, 2019). También cabe destacar que solo el $32,7 \%$ de los empleados encuestados han ascendido a pesar de que el $32,1 \%$ de ellos ha estado trabajando en el mismo puesto de trabajo durante 10 a 20 años y el 17,4\% de ellos ha estado trabajando durante más de 20 años. Podría ser consecuencia, según sus propios comentarios en la encuesta, de la falta de rutas de promoción.

Los resultados de la encuesta con respecto al desempeño laboral y la percepción de la gestión del talento [Gráfico 1] muestran que, en general, los empleados encuestados declaran sentirse bastante satisfechos con su desempeño laboral y se consideran muy competentes para desarrollar sus tareas. Sin embargo, cuando se les pregunta sobre ciertos aspectos del desempeño, sus opiniones difieren ligeramente. Por lo tanto, la mayoría de ellos sostienen que sus trabajos requieren tener iniciativa y que se necesita un desempeño creativo, así como también les permite mejorar sus habilidades profesionales. Sin embargo, hay menos empleados que consideran que tienen autonomía y libertad mientras realizan sus tareas, aún menos creen que pueden crecer personalmente en sus trabajos y, otros piensan que no tienen una buena formación interna.

Seguidamente, se preguntó a los empleados encuestados acerca de su percepción en la gestión de recursos humanos en su área. Esta batería de preguntas comienza preguntando sus opiniones sobre el modelo de proceso de selección actual a través de la Oferta Pública de Empleo. Las opiniones a este respecto están muy divididas. Sin embargo, la peor puntuación dentro de esta batería de preguntas se atribuye al proceso de promoción interna: 
solo 2,71 de 5, lo que probablemente esté influenciado por las escasas posibilidades de promoción mencionadas anteriormente y la falta de una carrera profesional adecuada a través del área de Turismo. Por otra parte, también se otorga una valoración muy baja al diseño de puestos de trabajo: solo 2,75 de 5 .

\section{Gráfica 1}

\section{VALORES MEDIOS DE LAS VARIABLES DEL CUESTIONARIO}

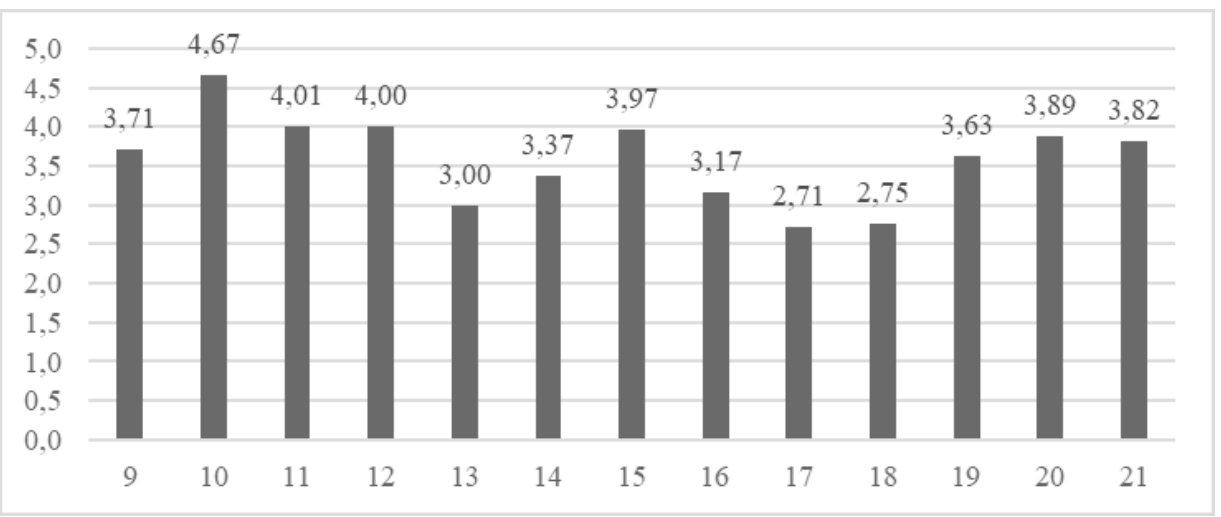

Fuente: Elaboración propia.

Finalmente, sí consideran necesaria una evaluación de desempeño en los términos establecidos por el EBEP y consideran bastante importante establecer un enfoque estratégico para sus trabajos. Para terminar, preguntados por su satisfacción en el trabajo, declaran, en general, estar muy satisfechos.

\section{GESTIÓN ESTRATÉGICA DEL TALENTO}

En este punto, se propone un conjunto de acciones para implementar con éxito la Gestión del Talento dentro de las organizaciones públicas en el área de Turismo definidas dentro del contexto de la Teoría del Valor (Sparrow y Makram, 2015).

En primer lugar, para crear valor, consideramos esencial para un progreso considerable en las organizaciones públicas contar con un grupo de candidatos talentosos. Actualmente, a las convocatorias de Oferta de Empleo Público acuden candidatos de forma masiva, sin embargo, no significa que la mayoría de los aspirantes tengan talento o sean lo suficientemente útiles para el puesto ofrecido. Para lograrlo, proponemos motivar a las personas con talento a que se incorporen a la función pública informando adecuadamente sobre el ejercicio y responsabilidad para con la sociedad que posee el empleado público: dar a conocer las normativas que rigen el desempeño, unificar dichas convocatorias en un mismo sitio web, proporcionar materiales gratuitos e informar que las evaluaciones del desempeño se llevan a cabo también dentro de las organizaciones públicas como elementos de evaluación de la productividad, por lo que conseguir un puesto de trabajo no implicase sólo la idea de obtener un trabajo para toda la vida. 
En segundo lugar, la importancia de capturar valor transformaría el sistema de acceso hacia uno que combine la evaluación de habilidades y destrezas del aspirante con pruebas que evalúen los procedimientos específicos del sector turístico, relegándolos a la última fase del proceso selectivo. Filtraría el masivo grupo de candidatos y actuaría como un predictor del desempeño laboral. Como ejemplo de buenas prácticas, se podrían tener en cuenta las evaluaciones basadas en habilidades que se llevan a cabo en los procesos de selección de la Unión Europea con resultados muy satisfactorios (European Personnel Selection Office, 2019).

En tercer lugar, una vez que los candidatos talentosos son empleados del sector público, se propone un proceso de tutela con el propósito apalancar el valor y agregar calidad al servicio en el que se incorporen. Actualmente, este proceso de mentorización se produce de manera breve y espontánea por parte de colegas, sin embargo, se sugiere sea planificado en términos de tiempo y contenido, así como de recompensas por los servicios de personal, oídas las diferentes organizaciones sindicales.

En cuarto lugar, se plantea proteger el valor de los efectivos mediante el desarrollo de rutas formativas a lo largo de la carrera profesional para contribuir a la especialización y actualización de conocimientos en el sector del turismo. El talento no debe considerarse inmutable, debe desarrollarse para seguir siendo útil a medida que cambian las necesidades de la institución. Del mismo modo, se recomienda desarrollar rutas de promoción bien diseñadas para retener a estos empleados preparados y talentosos. Teniendo en cuenta los resultados del cuestionario en nuestro caso de estudio, con esta propuesta, se intentaría paliar la falta de posibilidades para desarrollar una carrera profesional generadora de frustración entre los empleados de este sector.

En ese sentido, nuestra sugerencia descansa en la aplicación de evaluaciones del desempeño como parte del proceso de promoción junto a los concursos generales y específicos, por lo que los resultados positivos y negativos tendrían consecuencias.

El principal cambio se produciría, por un lado, en la promoción horizontal (promocionar sin cambiar del lugar de trabajo) donde se evalúe el desempeño como parte del proceso/ruta seleccionada y por otro, exigir a los empleados con resultados negativos en las evaluaciones del desempeño participar en los concursos internos generales para ser trasladados de su lugar de trabajo a otro, en un intento de mejorar su rendimiento.

Finalmente, con el objeto de seguir protegiendo el talento y evitar el presentismo, en la última etapa del conjunto de acciones, se apuesta por asegurar la lealtad de los empleados mediante lo que Thompson (2002) llama estrategia de Recompensa Total. Es decir, recompensas económicas y no económicas (horarios flexibles, servicio de salud, guardería, etc.) que pueden satisfacer a los empleados, generando fidelidad hacia la institución. No cabe duda, ambos tipos de recompensas tienen un costo para la organización, por lo que sugerimos compartir esa información con los miembros que la conforman. Otro factor clave directamente relacionado con la fidelidad es el salario emocional (desarrollo profesional, rol desafiante, libertad y autonomía en las tareas, crecimiento personal, reconocimiento de logros, sentimiento de pertenencia, etc.) que contribuye al bienestar de cada empleado cuidando de ellos personal y profesionalmente, como una forma indirecta de mejorar la competitividad y la productividad (Pérez-del-Prado, 2018). 
Por lo tanto, unir todas las piezas de esta propuesta podría ayudar a atraer y descubrir talento, mejorando el desempeño de la Administración Pública en la esfera del turismo a través de sus empleados más productivos.

\section{DISCUSIÓN Y CONCLUSIONES}

La importancia del turismo en el sector servicios en la economía española en general y, en la de Andalucía en particular, así como el papel clave de la Administración Pública en éste área, en un contexto cada vez más exigente y competitivo (Cuadrado-Roura y López-Morales, 2014), necesita atraer y retener a trabajadores versátiles y transversales con habilidades innatas que mantengan un desempeño excelente (Nijs et al., 2014) para mejorar el impacto positivo del turismo en la economía.

Actualmente, se detectan dos problemas en la gestión de empleados en las organizaciones públicas. Por un lado, se implantan diferentes sistemas de gestión de sus recursos humanos (Longo, 2004), sin planificar la atracción de talentos que hagan frente al nuevo entorno laboral y por otro, una falta de transferibilidad de las experiencias exitosas de la Gestión del Talento en las empresas privadas dedicadas al turismo (Alferaih et al, 2018; Li et al., 2018) a la Administración pública del sector. Además, el sector turístico en Andalucía adolece de una difusa información en el número de efectivos y acerca de las percepciones de sus trabajadores sobre el valor que pueden aportar a la organización y viceversa. Todo ello, dificulta la estrategia a seguir para incorporar una propuesta que implemente la Gestión del Talento de las instituciones que la integran.

Desde la perspectiva teórica, la Teoría del Valor facilita de manera analítica y simplificada un mecanismo desarrollado en cuatro etapas donde cimentar la Gestión del Talento (Sparrow y Makram, 2015). Proporciona un instrumento innovador al que adaptar la gestión de los recursos humanos en la Función Pública en estrecha relación con su particular contexto (Harsch y Festing, 2020). La gran dificultad a la que nos enfrentamos es la rigidez estructural que, hasta la última modificación estatutaria (Boletín Oficial del Estado, 2015), no permitía cambios considerables en la gestión de sus empleados. No obstante, la implantación de las diferentes fases nos acerca a elementos comunes entre ambos sectores, público y privado, ya sea el diseño estratégico de los puestos de trabajo, la promoción interna o la capacitación especializada frente a los nuevos retos.

Es por esto, se ha propuesto una batería de acciones que vinculan la base teórica de la Gestión del Talento con los resultados obtenidos en el cuestionario piloto realizado a una muestra del conjunto de los trabajadores públicos del sector turístico de Andalucía. La radiografía organizacional lograda a través de la aproximación al número real de trabajadores, al examen de su estructura y la recogida de información sobre las percepciones de sus trabajadores acerca de la gestión de los recursos humanos mediante un cuestionario, podría ser el punto de partida para profundizar en el papel de los empleados del sector público en diferentes áreas, sin importar que, en este trabajo, solo se haya aplicado al turismo.

A pesar de que el objetivo principal del cuestionario fue su validación, se han llegado a algunas conclusiones interesantes. En particular, aquellos relacionados con la forma en que se sienten en sus puestos de trabajo, si pueden crecer personal y profesionalmente y si pueden desarrollar sus habilidades. En este sentido, las principales preocupaciones de los 
empleados del sector público encuestados fueron la formación, el sistema de acceso y el diseño de los puestos de trabajo, etapas clave en la generación y retención de valor. Asimismo, se han propuesto más acciones en otros ejes como la evaluación del desempeño o la promoción, pues entendemos que las políticas de Gestión del Talento no se pueden llevar a cabo independientemente como si de áreas estancas se trataran, habiendo de considerarse como una estrategia integral para mejorar la ventaja competitiva de una organización.

$\mathrm{Al}$ respecto, se ha enfatizado la necesidad de realizar cambios en los métodos de acceso como primer paso para atraer y seleccionar personas con talento. También se ha considerado necesario cuidar a los empleados públicos mediante la consideración de incentivos basados en resultados positivos en la evaluación del desempeño o intentar motivar aquellos con resultados negativos al cambiar de puesto de trabajo participando obligatoriamente en los concursos internos. De igual modo, se propone fortalecer la retención introduciendo recompensas económicas y no económicas, considerándolas desde dos perspectivas: 1) para mejorar el bienestar de los empleados y 2) como una forma de fidelizarlos a la institución.

Por lo tanto, es una cuestión de urgencia implementar políticas de Gestión del Talento de manera efectiva en las organizaciones del sector público como una forma de enfrentar el dinamismo típico de sectores como el de Turismo y considerando sus impactos directos e indirectos en la economía de regiones como Andalucía. Es necesario eliminar el problema de la inflexibilidad, el bajo rendimiento e improductividad dentro de las Administraciones y consideramos que ahora es el momento de hacerlo dado que, a medio plazo, se producirá un importante relevo generacional y el mercado laboral y la Función Pública deben estar preparados para enfrentarse con éxito a una nueva realidad inestable y dinámica.

Finalmente, se han detectado dos limitaciones a partir de la aplicación del pretest: 1) desequilibrio, entre el marco teórico y la elaboración de preguntas, relacionado con la conciliación familiar y la rotación; 2) el envío online del cuestionario no ha resultado suficientemente ágil ni motivador para la obtención de respuestas completas, motivo por el que se tuvo que incidir telefónicamente, sobre todo, en pequeños núcleos rurales. Sin embargo, gracias a esta prueba a pequeña escala (Santos et. al, 2003 y Cea, 2009) estamos en disposición, en futuras investigaciones, de aproximar las necesidades y preparación de entrevistadores, los costes del trabajo de campo y la inclusión de las dos dimensiones del marco teórico; así como añadir técnicas cualitativas, como los grupos de discusión (Baelo y Haz, 2019), como medida complementaria del estudio exploratorio.

\section{AGRADECIMIENTOS}

F. Sánchez-Cubo disfruta de un contrato predoctoral para personal investigador en formación en el marco del Plan Propio de I+D+i de la Universidad de Castilla-La Mancha, cofinanciado por el Fondo Social Europeo (FSE).

\section{BIBLIOGRAFÍA}

ALFERAIH, A. (2017): «Developing a conceptual model to measure talent's turnover intention in tourism organisations of Saudi Arabia», International Journal of Organizational Analysis, vol. 25 (1), pp. 2-23. 
ALFERAIH, A., SARWAR, S., y EID, A. (2018): «Talent turnover and retention research: The case of tourism sector organisations in Saudi Arabia», Evidence-based HRM, vol. 6 (2), pp. 166-186.

ALPARSLAN, A. y SANER, T. (2020): «The influence of sustainable talent management on job satisfaction and organizational commitment: Moderating role of in-service training», Revista de Cercetare si Interventie Sociala, $\mathrm{n}^{\circ}$ 69, pp. 96-121.

BAELO, M. y HAZ, F.E. (2019): Metodología de investigación en ciencias sociales y jurídicas. Valencia, Tirant Humanidades.

BARRON, P. (2008): «Education and Talent Management: Implications for the hospitality industry», International Journal of Contemporary Hospitality Management, vol. 20 (7), pp. 730-742.

BHARWANI, S., y TALIB, P. (2017): «Competencies of hotel general managers: a conceptual framework», International Journal of Contemporary Hospitality Management, vol. 29 (1), pp. 393-418.

BLANCO, A. (2016): Liderazgo creador. Inteligencia actitudinal, racionalidad y diseño humano en la Administración. Madrid, Serie Innovación Social, INAP.

BRANDENBURGER, A., y NALEBUFF, B.J. (1995): «The right game: Use game theory to shape strategy», Harvard Business Review, vol. 73 (4), pp. 57-71.

BRATTON, J. y WATON, S. (2018): «Talent Management, emotional labour and the role of line managers in the Scottish hospitality industry: A roundtable discussion», Worldwide Hospitality and Tourism Themes, vol. 10 (1), pp. 57-68.

BOLETÍN OFICIAL DE LA JUNTA DE ANDALUCÍA (2008): «Decreto 43/2008 de 12 de febrero, regulador de las condiciones de implantación y funcionamiento de campos de golf en Andalucía», 12 de febrero de 2008, n 41, pp. 9-16.

BOLETÍN OFICIAL DEL ESTADO (1985): «Ley 7/1985, de 2 de abril, Reguladora de las Bases del Régimen Local», 3 de abril de 1985, nº 80, pp. 8945-8964.

BOLETÍN OFICIAL DEL ESTADO (2010): «Ley 5/2010, de 11 de junio, de Autonomía Local de Andalucía», 19 de julio de 2010, n 174, pp. 63395-63451.

BOLETÍN OFICIAL DEL ESTADO (2011): «Ley 13/2011, de 23 de diciembre, del Turismo de Andalucía», 20 de enero de 2012, n 17, pp. 4732-4771.

BOLETÍN OFICIAL DEL ESTADO (2015): «Real Decreto Legislativo 5/2015, de 30 de octubre, por el que se aprueba el texto refundido de la Ley del Estatuto Básico del Empleado Público», 30 de octubre de 2015, n 261, pp. 103105-103159.

CEA, M.A. (2009): Metodología cuantitativa. Estrategias y técnicas de investigación social. Madrid, Síntesis Sociología.

CHATAIN, O., y ZEMSKY, P. (2011): «Value creation and value capture with frictions»., Strategic Management Journal, vol. 32, pp. 1.206-1.231.

CONSEJERÍA DE TURISMO Y DEPORTE (2017): Balance del Año Turístico en Andalucía. Disponible en http://cort.as/-MntO Consulta el 25 de febrero de 2019.

CUADRADO-ROURA, J.R., y LÓPEZ-MORALES, J.M. (2014): «El turismo, motor del crecimiento y de la recuperación de la economía española», Estudios Turísticos, $\mathrm{n}^{\mathrm{o}}$ 200, pp.- 19-38. 
DEERY, M. (2008): «Talent Management, work-life balance and retention strategies», International Journal of Contemporary Hospitality Management, vol. 20 (7), pp. 792-806.

DEERY, M., y JAGO, L. (2015): «Revisiting Talent Management, work-life balance and retention strategies», International Journal of Contemporary Hospitality Management, vol. 27 (3), pp. 453-472.

DELFGAAUW, J., y DUR. R. (2010): «Managerial Talent, Motivation, and Self-Selection into Public Management», Journal of Public Economics, vol. 94 (9/10), pp. 654-660.

EMPRESA PÚBLICA PARA LA GESTIÓN DEL TURISMO Y EL DEPORTE DE ANDALUCÍA, S.A. (2019) Web de Turismo Andaluz - Solicita información turística. Disponible en http://www.andalucia.org/es/contacta-con-nosotros/busqueda/rapida/ Consulta el 10 de marzo de 2019.

ESIC. Marketing \& Business School \& Praxis MMT Corporation (2017): «El Informe "Young Business Talents"». Disponible en https://www.youngbusinesstalents.com/ wp-content/uploads/2018/06/The-YBT-Report_Spain_Espan\%CC\%83ol-2018.pdf Consulta el 2 de marzo de 2019.

EUROPEAN PERSONNEL SELECTION OFFICE (2019): «How to apply - Sample tests». Disponible en https://epso.europa.eu/how-to-apply/sample-tests Consulta el 3 de marzo 2019.

FORNELL, C. y LARCKER, D.F. (1981): «Evaluating Structural Equation Models with Unobservable Variables and Measurement Error», Journal of Marketing Research, vol. 18 (1), pp. 39-50.

GAD3, Educa 20.20 y Fundación AXA (2017): «El futuro laboral de los jóvenes. Presentación de resultados nacionales». Disponible en https://www.axa.es/documents/1119421/134089346/E2020+Secundaria+Presentaci\%C3\%B3n+Espa\%C3\% B1a.pdf/53302f98-cd6d-cff4-3ddc-10a3b2ec812e Consulta el 2 de marzo de 2019.

HARSCH, K. y FESTING, M. (2020): «Dynamic talent management capabilities and organizational Agility: A qualitative exploration», Human Resource Management, vol. 59 (1), pp. 43-61.

HAUSKNECHT, J.P., RODDA, J. y HOWARD, M.J. (2009): «Targeted employee retention: Performance-based and job-related differences in reported reasons for staying», Human Resource Management, vol. 48 (2), pp. 269-288.

INSTITUTO ANDALUZ DE ADMINISTRACIÓN PÚBLICA (2008): «Análisis de los puestos de Atención Directa a la Ciudadanía». Sevilla. Consejería de Justicia y Administración Pública. Dirección General de Inspección y Evaluación.

INSTITUTO DE ESTADÍSTICA Y CARTOGRAFÍA DE ANDALUCÍA (2017): «Sistema de Información Multiterritorial de Andalucía (SIMA) ». Disponible en https://www. juntadeandalucia.es/institutodeestadisticaycartografia/sima/index2.htm Consulta 6 de marzo de 2019.

INSTITUTO NACIONAL DE ESTADÍSTICA (2018): «Cuenta Satélite del Turismo». Disponible en https://www.ine.es/dyngs/INEbase/es/operacion.htm?c=Estadistica_C\& cid $=1254736169169 \&$ menu $=u l t i D a t o s \& i d p=1254735576863$ Consulta el 3 de marzo de 2019. 
INSTITUTO NACIONAL DE ESTADÍSTICA (2019): «Encuesta de Población Activa». Disponible en http://cort.as/-9vMz Consulta el 3 de marzo de 2019.

JAUHARI, V. y SANJEEV, G. M. (2012): «Responding to the emerging strategic and financial issues in the Indian hospitality industry», Worldwide Hospitality and Tourism Themes, vol. 4 (5), pp. 478-485.

JOOSS, S., MCDONNELL, A., BURBACH, R. y VAIMAN V. (2019): «Conceptualising talent in multinational hotel corporations», International Journal of Contemporary Hospitality Management, vol. 31 (10), pp. 3.879-3.898.

KING, K.A. y VAIMAN, V. (2019): «Enabling effective talent management through a macro-contingent approach: A framework for research and practice», Business Research Quarterly, vol. 22 (3), pp. 194-206.

LI, Z., DUVERGER, P. y YU, L. (2018): «Employee creativity trumps supervisor-subordinate guanxi: Predicting prequitting behaviors in China's hotel industry», Tourism Management, vol. 69, pp. 23-37.

LONGO, F (2004): Mérito y flexibilidad. Las gestión de las personas en las organizaciones del sector público. Barcelona, Ediciones Paidós Ibérica, S.A.

LOON, M., OTAYE-EBEDE, L. y STEWART, J. (2020): «Thriving in the New Normal: The HR Microfoundations of Capabilities for Business Model Innovation. An Integrated Literature Review», Journal of Management Studies, vol. 57 (3), pp. 698726.

MA, C., REN, L., CHEN, P. y HU, R.X. (2019): «Institute-Hotel Coordinating Barriers to Early Career Management-Hoteliers' Accounts 2019», Journal of China Tourism Research, vol. 16 (2), pp. 297-317.

MAKAREM, Y., METCALFEB, B.D. Y AFIOUNIA, F. (2019): «A feminist poststructuralist critique of talent management: Toward a more gender sensitive body of knowledge», Business Research Quarterly, vol. 22, pp. 181-193.

MAXWELL, G.A. y MACLEAN, S. (2008): «Talent Management in hospitality and tourism in Scotland: Operational implications and strategic actions», International Journal of Contemporary Hospitality Management, vol. 20 (7), pp. 820-830.

MONDÉJAR, J. (2016): «Análisis multivariante: diseño de encuestas». En. J.L. Sánchez (Presidencia). Jornada de Investigación Turística. Universidad de Málaga. España.

NIJS, S., GALLARDO-GALLARDO, E., DRIES, N. y SELS, L. (2014): «A multidisciplinary review into the definition, operationalization, and measurement of talent», Journal of World Business, vol. 49 (2), pp. 180-191.

NUNNALLY, J.C. y BERNSTEIN, I.H. (1994): Psychometric theory (3rd ed.). New York, McGraw-Hill, Inc.

PAULI, U. y POCZTOWSKI, A. (2019): «Talent management in SMEs: An exploratory study of Polish companies», Entrepreneurial Business and Economics Review, vol. 7 (4), pp. 199-218.

PÉREZ-DEL-PRADO, D. (2018): «¿Qué es el salario emocional? hacia nuevas fórmulas de acción social empresarial». Revista de Información Laboral, $\mathrm{n}^{\circ}$ 1, pp. 235-254.

RICOU, J. (2018): "Fiebre por ser funcionario". La Vanguardia. Recuperado de: https:// www.lavanguardia.com/vida/20180305/441278991556/funcionario-empleo-publicodemanda.html. 
ROSADO, D.P., ROMÃO, A., LOPES, H.S.C., BALTAZAR, M.S. y FONSECA, D. (2019): «Strategic management and retention of talent: Challenges in the Portuguese Army», Journal of Reviews on Global Economics, vol. 8, pp. 130-140.

SANTOS, J., MUÑOZ, A., JUEZ, P. y CORTIÑAS, P. (2003): Diseño de encuestas para estudios de mercado. Técnicas de muestreo y análisis multivariante. Madrid, Ed. Centro de Estudios Ramón Areces, S.A.

SAVOV, R., LANCARIC, D. y KOZAKOVA, J. (2020): «Size of the Company as the Main Determinant of Talent Management in Slovakia», Journal of Risk and Financial Managemen, vol. 13 (3), pp. 1-14.

SPARROW, P.R. y MAKRAM, H. (2015): «What is the value of talent management? Building value-driven processes within a talent management architecture», Human Resource Management Review, vol. 25 (3), pp. 249-263.

SUNDBO, J. (2009): «Innovation in the experience economy: a taxonomy of innovation organisations», The Service Industries Journal, vol. 29 (4), pp. 431-455.

STIRLING, W.C. (2011): Theory of Conditional Games. Cambridge, Cambridge University Press.

TANSLEY, C. (2011): «What do we mean by the term "talent" in Talent Management?», Industrial and Commercial Training, vol. 43 (5), pp. 266-274.

THOMPSON, P. (2002): Total Reward. London, Chartered Institute of Personnel and Development,

WERTS, C.E., LINN, R.L. y JORESKOG, K.G. (1974): «Intraclass reliability estimates - Testing structural assumptions», Educational and Psychological Measurement, vol. 34 (1), pp. 25-33.

WORLD TRAVEL y TOURISM COUNCIL (2019): «Travel \& Tourism Economic Impact 2019». Disponible en https://www.wttc.org/-/media/files/reports/economic-impactresearch/regions-2019/world2019.pdf Consulta 10 de marzo 2019.

ZHANG, S. y BRIGHT, D. (2012): «Talent definition and Talent Management recognition in Chinese private-owned enterprises», Journal of Chinese Entrepreneurship, vol. 4 (2), pp. 143-163. 


\section{ANEXO}

\section{GESTIÓN DE RECURSOS HUMANOS}

Le damos la bienvenida al cuestionario sobre planificación de Recursos Humanos en los empleados públicos del Área de Turismo. La información que nos proporcione se tratará de forma CONFIDENCIAL Y ANÓNIMA y no será utilizada para ningún propósito distinto a la elaboración de un estudio de investigación de la Universidad de Málaga sobre la gestión de personal del colectivo mencionado.

Consta de 21 preguntas divididas en tres secciones: datos socio-laborales, percepción del desempeño laboral y gestión de los RRHH. Utilice el espacio final para cualquier comentario respecto a este cuestionario. El tiempo estimado de respuesta es de 5 minutos.

\section{Datos socio-laborales}

1. Género

Hombre

2. Edad

a. Menos de 26

b. 26 a 35

c. 36 a 45

d. 46 a 55

e. Más de 55

3. Indique donde trabaja

a. Delegación de Turismo de la Junta de Andalucía

b. Ayuntamiento. Área de Turismo

c. Diputación. Área de Turismo

4. Indique la zona donde trabaja

a. Capital

b. Población costera

c. Población de interior
5. ¿Cuánto tiempo lleva trabajando en el área de Turismo de su Institución?
a. Menos de 6 meses
b. De 6 meses a 2 años
c. De 2 años a 5 años
d. De 5 años a 10 años
e. De 10 años a 20 años
f. Más de 20 años

6. ¿Qué nivel de estudios ha finalizado?

a. Educación secundaria/Bachiller

b. Formación Profesional Grado Medio

c. Formación Profesional. Grado Superior

d. Estudios universitarios de Grado

e. Estudios universitarios de Máster

f. Estudios universitarios. Doctor

g. Otros

7. ¿Ha ascendido desde que accedió al Área de Turismo?
a. $\mathrm{Si}$

b. No

8. ¿Qué relación laboral tiene?

a. Funcionario de carrera

b. Laboral fijo

c. Laboral temporal

Cómo percibe su desempeño laboral

Marque de menos (1 muy desacuerdo) a más (5 totalmente de acuerdo) según su valoración en cada cuestión.

9. ¿Considera que tiene autonomía y libertad en el desarrollo de las tareas?

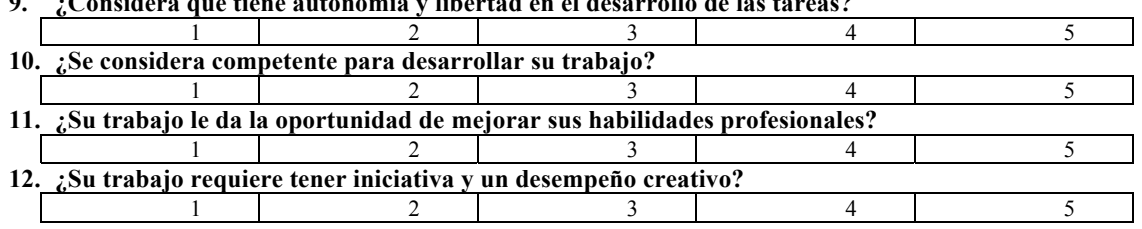


13. ¿Considera que la institución le oferta una buena formación acorde a su trabajo?

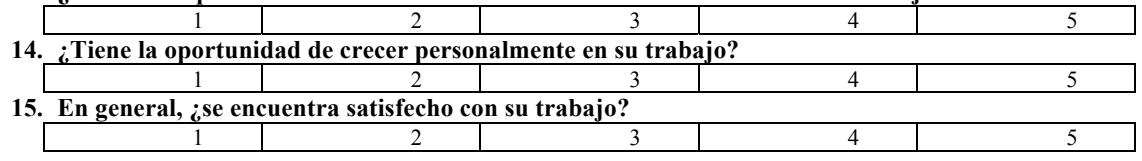

Cómo percibe la gestión de los recursos humanos en su Institución

Marque de menos (1 muy desacuerdo) a más (5 totalmente de acuerdo) según su valoración en cada cuestión.

16. Considero acertado el modelo de selección de personal en la Oferta de Empleo Público

\begin{tabular}{|c|c|c|c|c|}
\hline 1 & 2 & 3 & 4 & 5 \\
\hline 17. Considero adecuado el modelo de promoción interna & 3 & 4 & 5 \\
\hline 1 & 2 & 3 & 4 & 5 \\
\hline
\end{tabular}

18. Creo ajustado el diseño de los puestos de trabajo

\begin{tabular}{|c|c|c|c|c|}
\hline 1 & 2 & 3 & 4 & 5 \\
\hline 19. Opino que es necesaria la evaluación del desempeño en los términos que establece el Estatuto Básico del
\end{tabular} Empleado Público (EBEP)

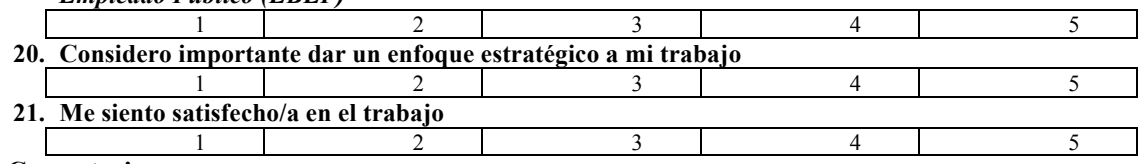

Comentarios 